\title{
RESISTÊNCIA MECÂNICA DE TIJOLOS ADOBE REFORÇADOS COM RESÍDUO DE CASCA DE OVO
}

\author{
Mechanical strength of adobe bricks reinforced with egg shell waste \\ Rafael Rodrigues da Rocha ${ }^{1}$, Gustavo Henrique Vieira Barros ${ }^{2}$, Renan Jhoni da \\ Silva $^{2}$, Rebeca Delatore Simões ${ }^{1}$ \\ ${ }^{1}$ Universidade Estadual Paulista - UNESP, Faculdade de Ciências e Engenharia, Câmpus \\ de Tupã, SP. ${ }^{2}$ Escola Técnica Estadual (ETEC) Prof. Massuyuki Kawano, Tupã, SP. \\ E-mail: $\quad$ rafael.rodrigues2001@outlook.com; gusbarros378@gmail.com; \\ renanjh7@gmail.com; rebeca.simoes@unesp.br
}

RESUMO - O setor da construção civil impacta diretamente no desenvolvimento de uma nação. Em contrapartida, consome uma grande quantidade de energia, recursos naturais e o setor com maior descarte de resíduos. A preocupação com o impacto ambiental causado pelo desenvolvimento desse campo tem sido alvo de diversas pesquisas. Grande parte dos estudos convergem para a reciclagem dos resíduos gerados e reinserção, destes materiais, no ciclo construtivo. Neste trabalho investigou-se a perda de umidade e a resistência à compressão mecânica de adobes produzidos com argamassa reciclável, resíduos de casca de ovo triturada e hidratados com diferentes dosagens de vinhaça e água. Os materiais foram processados manualmente, prensados em prensa hidráulica e curados por 7 e 14 dias. Foi realizado o estudo da granulometria da matéria prima triturada. Durante o processo de cura foi estudado a perda de umidade. Os corpos de prova foram ensaiados por medidas de resistência à compressão mecânica. Os resultados de perda da umidade e dos testes mecânicos apresentaram, para um dos traços propostos, valores próximos aos padrões requeridos pela Associação Brasileira de Normas Técnicas, indicando que estes resíduos podem ser uma alternativa promissora para fabricação de tijolos e contribuir com a redução do impacto ambiental e economia de recursos hídricos.

Palavras-chave: Argamassa reciclável; Casca de ovo; Vinhaça.

ABSTRACT - The civil construction sector directly impacts the development of a nation. On the other hand, it consumes a large amount of energy, natural resources and the sector with the highest waste disposal. The concern with the environmental impact caused by the development of this field has been the subject of several researches. Most of the studies converge on the recycling of waste generated and the reinsertion of these materials in the construction cycle. In this work we investigated the loss of moisture and the resistance to mechanical compression of adobes produced with recyclable mortar, crushed eggshell residues and hydrated with different dosages of vinasse and water. The materials were processed manually, pressed in a hydraulic press and cured for 7 and 14 days. The study of the granulometry of the crushed raw material was carried out. During the curing process, moisture loss was studied. The specimens were tested by measures of resistance to mechanical compression. The results of moisture loss and mechanical tests showed, for one of the 
proposed features, values close to the standards required by the Brazilian Association of Technical Standards, indicating that these residues can be a promising alternative for the manufacture of bricks and contribute to reducing the impact environmental and water resource savings.

Keywords: Recyclable mortar; Eggshell; Vinasse.

\section{INTRODUÇÃO}

A ampla divulgação de problemas ambientais ocorridos e de movimentos ecológicos propiciou uma mudança no cenário de produção das empresas, que perceberam a necessidade de melhorar o desempenho ambiental, através de modificações em seus processos produtivos com a adoção das chamadas tecnologias limpas para obter sucesso em seus negócios (DIAS, 2017a).

A indústria da construção civil tem papel importante no desenvolvimento econômico e social de um país, sendo que fatores como a qualidade de vida e infraestrutura estão diretamente ligadas a esse setor, além do grande impacto no Produto Interno Bruto (PIB) de um país e no PIB mundial. Entretanto, mesmo sendo considerada como uma das mais importantes atividades industriais, o setor é considerado um dos maiores agentes de degradação do meio ambiente, consumindo grande quantidade de energia, recursos naturais finitos e alta geração de resíduos (TAM et al., 2018).

O setor gera grande quantidade de resíduos que, em grande maioria, são depositados em locais irregulares. Por esses motivos, é uma área que os órgãos regulamentadores estão sempre atentos ao consumo desenfreado de matérias primas e sempre desenvolvendo novas tecnologias visando a economia e reutilização de matérias (KLEPA, 2019.

A reciclagem é, dentre outras, uma alternativa que pode ser desenvolvida para minimizar os problemas causados pela exploração desmedida do meio ambiente. Ela promove uma alternativa para reduzir o consumo de matéria prima, a geração desordenada de resíduos e os problemas associados como o crescimento de vida útil dos aterros, diminuição de pontos de descartes clandestinos, e redução nos custos de gerenciamento de resíduos (BRASILEIRO; MATOS, 2015).

Esta pesquisa foi motivada pela abundância dos resíduos vinhaça e casca de ovo encontrados na região do centro oeste do interior paulista (Alta Paulista). A região é rodeada de usinas sucroenergéticas. Também, é conhecida como a capital nacional do ovo e conta com uma usina de reciclagem de resíduos da construção civil que coleta resíduos recicláveis e reutilizáveis em mais de $70 \%$ da área urbana das cidades do seu entorno. Portanto, o foco principal desta pesquisa experimental foi estudar um processo de fabricação de tijolos ecológicos, produzidos com argamassa reciclável, casca de ovo e vinhaça, utilizando o método "adobe", de modo que, o produto final obtido atendesse os requisitos, de resistência a compressão mecânica para tijolos de solo-cimento, recomendados pela Associação Brasileira de Normas Técnicas (ABNT).

\section{REVISÃO DA LITERATURA}

Com a urbanização acelerada, que resultou no rápido adensamento das cidades, e, por consequência, o crescimento das atividades do setor construtivo, além da larga exploração dos recursos naturais, a geração de resíduos da construção e demolição (RCD) alcançou índices alarmantes, produto do desperdício nas obras de construções, reformas e demolições.

Estima-se que em torno de $35 \%$ das quantidades geradas RCD do mundo sejam direcionadas para aterros, sem nenhum tratamento adicional, embora esforços para reciclar e reutilizar sejam cada vez mais investigados (MENEGAKI; DAMIGOS, 2018). A falta de gerenciamento adequado desses materiais afeta as cidades nos aspectos sociais, econômicos e ambientais (BRASILEIRO; MATOS, 2015).

A tarefa de quantificação em países subdesenvolvidos é uma tarefa difícil, pois uma importante fonte na geração de RCC são os geradores informais, e podem representam uma parcela importante dos RCCs gerados em um município. Porém, segundo a ABRELPE (Associação Brasileira de Empresas de Limpeza Pública e resíduos especiais), só no ano de 2018, - RCD recolhido nas cidades brasileiras 
representa cerca de $40 \%$ da massa total de RSU gerados neste ano. (ABRELPE, 2019).

Visando regulamentar a gestão diferenciada dos resíduos, em 2002 foi instituída a Resolução no 307 do Conselho Nacional do Meio Ambiente (BRASIL,2002) que estabelece diretrizes, critérios e procedimentos para a redução, o reaproveitamento e a reciclagem dos RCCs, dividindo-os em quatro classes A, B, C e D. Isso ocorreu a fim de evitar os desperdícios e salvaguardar os recursos naturais e o meio ambiente para as gerações futuras, mediante a promoção de um desenvolvimento sustentável.

A classe A são os materiais que podem ser reciclados/reutilizados como agregado em obras de infraestrutura, edificações e canteiro de obras como Tijolos, telhas e revestimentos cerâmicos; blocos e tubos de concreto e argamassa. Na B, estão os materiais que podem ser reciclados e ganhar outras destinações como vidro, gesso, madeira, plástico e papelão. A classe $C$, é composta por itens para o qual não existe ou não é viável aplicação econômica para recuperação ou reciclagem como tecidos e lixas desde que não tenham contato com substância que o classifique como D. Na classe $D$ são aqueles materiais nocivos ou que tiveram contato com substâncias nocivas à saúde, como exemplo, solvente e tintas; telhas e materiais de amianto e entulho de reformas em clínicas.

$\mathrm{Na}$ indústria da construção civil, além da iniciativa da criação das usinas de reciclagem (MARQUES et al. , 2020) já se tornou usual reciclar diversos resíduos para fabricação de novos produtos para esse mercado, reduzindo o consumo de matéria prima finita, como a artefatos de cimento desenvolvido com PET reciclada (NEVES et al., 2020; TOSELLO et al., 2021), fibras naturais (GRILLO; SARON, 2020; NEVES et al., 2020, MOTA et al., 2020) com borracha vulcanizada (SILVA et al. ,2017) e com a reutilização do próprio resíduo de construção civil, em especial a argamassa, usualmente conhecida como reboco (REIS, 2016; SHARKAWI, 2016).

A vinhaça é produzida como subproduto da produção de álcool. Para a produção de 1 litro de álcool destilado é necessário gerar de 13 a 18 litros de vinhaça (ROSSETTO, 1987). Devido à alta quantidade de matéria orgânica, é comumente utilizada como fertilizante agrícola. Entretanto, quando depositada em quantidades superiores à capacidade de retenção de íons do solo pode apresentar consequências inestimáveis. Em quantidades desbalanceadas, saliniza o solo, é considerada altamente nociva à fauna, flora, microfauna e microflora das águas doces, e afugenta a fauna marinha que vem à costa brasileira para procriação (SILVA; GRIEBELER; BORGEET, 2007).

A casca do ovo de galinha é um composto biocerâmico, permeável aos gases a água, cuja função é proteger e fornecer o cálcio necessário a plena formação do seu conteúdo interno. Sua camada calcária é composta por uma rede de fibras proteicas composta por cristais de carbonato de cálcio ( $96 \%$ do peso da casca), carbonato de magnésio (1\%) e fosfato de cálcio (1\%) (NEVES, 1998). Além do Cálcio, mineral mais abundante, outros minerais podem ser encontrados em menores concentrações, como magnésio $(\mathrm{Mg})$, estrôncio $(\mathrm{Sr})$, ferro $(\mathrm{Fe})$, selênio (Se), entre outros (MILBRADT et al., 2015).

A indústria de avicultura de postura comercializa diversas versões do produto como os ovos em pó, líquidos, congelados e os específicos para panificação proporcionando vantagens econômicas, extensão da vida útil do produto e facilidades no transporte, porém, gera um número expressivo de resíduos de cascas de ovo.

O tijolo de adobe é definido como o bloco de barro, moldado em forma artesanal e seco ao ar, sem passar pelo processo de queima. É um dos mais antigos materiais de construção utilizados no mundo, sabe-se quase $30 \%$ da população total do mundo ainda reside em casas de barro. As características físicas e/ou mecânicas do tijolo de adobe pode ser melhorada com a incorporação de elementos estabilizantes como as fibras naturais (NEVES et al., 2020).

Do ponto de vista sustentável, o adobe possui características positivas, pois tem a vantagem de ser um elemento reutilizável, quando não cozido, pode ser triturado e umedecido para voltar ao estado original. Sua produção não necessita de grande quantidade de energia e ainda é um excelente isolante térmico, mantendo a temperatura dos ambientes sempre balanceados. A desvantagem do adobe é que suas construções precisam ser protegidas da umidade, podendo absorver até 30 vezes mais umidade do que o tijolo cozido (DIAS, 2017b). Assim, é importante conhecer os materiais recicláveis para a produção de adobes e aplicação em alvenaria, além dos métodos de ensaio para sua caracterização física e mecânica. 


\section{METODOLOGIA}

O solo do tipo argissolo foi coletado na área experimental da FCE-UNESP, a argamassa reciclável foi coletada em caçambas da Usina de reciclagem de resíduos da construção civil do município de Tupã, região da Alta Paulista, centro oeste do Estado de São Paulo. A vinhaça e a casca de ovo foram doadas por uma usina de açúcar e álcool e uma empresa avícola de postura respectivamente. O cimento utilizado foi o CP II 32 (Votoran).

Além do traço piloto, foram fabricados mais 4 lotes, com diferentes proporções em massa de solo, cimento (C), cal, argamassa reciclável (AR) e casca de ovo (CO) conforme apresenta a Tabela 1. A água e a vinhaça (V), em diferentes dosagens, foram usadas como agente hidratante. Eles foram adicionados em quantidade mínima suficiente para agir como ligante dos materiais secos. Para a produção dos corpos de prova do traço piloto (lote A) foi escolhido inicialmente o traço, solo/cimento $1: 10$, comumente utilizado na região da Alta Paulista.

Tabela 1. Traços - \% dos componentes

\begin{tabular}{rrrrrrr}
\hline Lote & Solo & \multicolumn{1}{c}{ C } & AR & Cal & CO & Água/V \\
\hline & $(\%)$ & $(\%)$ & $(\%)$ & $(\%)$ & $(\%)$ & $(\% / \%)$ \\
\hline A & 90 & 10 & 0 & 0 & 0 & $100 / 0$ \\
\hline B & 85 & 10 & 0 & 5 & 0 & $0 / 100$ \\
\hline C & 65 & 5 & 25 & 3 & 2 & $0 / 100$ \\
\hline D & 65 & 5 & 25 & 3 & 2 & $25 / 75$ \\
\hline E & 65 & 5 & 25 & 3 & 2 & $50 / 50$ \\
\hline
\end{tabular}

Fonte: Os autores.

No processo de fabricação fez-se uma mistura manual dos materiais a serem prensados, onde esta mistura é depositada em uma forma retangular, fabricada com redução de $30 \%$ do tamanho padrão comercial. Nela contém um molde vazado de alumínio com dimensões adequadas para facilitar a remoção do material e melhorar o aspecto do acabamento externo do produto final.

Para o procedimento de otimização da fabricação dos adobes via prensagem foi utilizada uma prensa hidráulica manual, com capacidade máxima de carga de 50 toneladas. Para cada lote (A, B, C, D e E) foram fabricados 6 corpos de prova (cp) nas dimensões $6,0 \mathrm{~cm}$ (comprimento) $\times 3,0 \mathrm{~cm}$ (altura) $\times 4,5 \mathrm{~cm}$ (largura). Destes $6 \mathrm{cps}$, 3 foram ensaiados por compressão mecânica com 7 dias de cura e 3 com 14 dias de cura. Os corpos de prova foram curados na sombra, em ambiente coberto, livre de umidade até o período de 14 dias. A Figura 1 ilustra a sequência (mistura, moldagem e cura) do processo de fabricação dos adobes.

Figura 1. Fabricação: mistura (a), moldagem por prensagem (b), e cura (c)

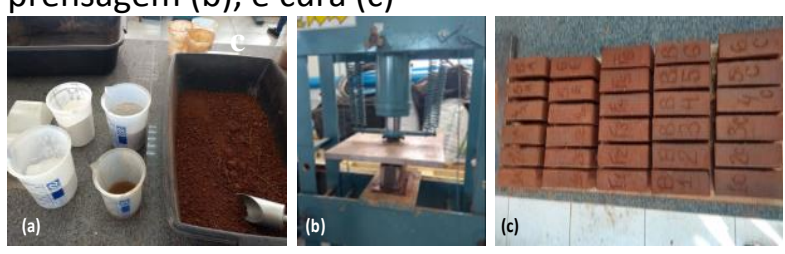

Fonte: Os autores.

A caracterização da matéria prima já triturada (solo, argamassa reciclável e casca de ovo) foi realizada através do ensaio de granulometria, em conformidade com a NBR 7181/1984 - Método de Ensaio da ABNT. O ensaio foi conduzido utilizando as peneiras de números: $4,8,16,30,50,100$ e 200 com suas respectivas aberturas em milímetros: 4,760; 2,$380 ; 1,190 ; 0,590 ; 0,297 ; 0,149$ e 0,074 . As porcentagens retidas nas peneiras foram determinadas através da razão entre a massa retida na peneira desejada e a massa total ensaiada.

Por utilizar uma prensa manual para a fabricação dos tijolos, que pode causar possíveis variações no peso final dos corpos de prova, realizaram-se as aferições diárias das massas de todos os corpos de prova (tijolos) analisados nesse experimento, durante todo o processo de cura (7 e 14 dias), em balança digital, com precisão 1,0 gr.

As medidas de compressão mecânica foram realizadas em conformidade com as especificações das normas ABNT NBR 8491/12 e 8492/12, prensa UTS ADL 2000/ EL Digital (United Test Malaysia) com certificado de calibração INTERMETRIC, no laboratório da Empresa G10 Concreto, no município de Tupã / SP.

\section{RESULTADOS E DISCUSSÃO Granulometria}

Conhecer a composição granulométrica dos componentes que formarão o adobe é um fator preponderante para obter peças como boa resistência mecânica e durabilidade e baixa retração. A granulometria de um solo tem influência direta na qualidade e no custo do solocimento (SOUZA, 2008). Segantini (2000) considera importante a presença de materiais mais grossos, com função apenas de enchimento, 
pois haverá liberação de maiores quantidades de cimento para aglomerar os grãos menores.

O ensaio para a determinação da composição granulométrica do argissolo apresentou $23 \%$ de material fino, grãos com diâmetros entre 0,060 e 0,200 mm, 54,3\% grãos acima de 0,297 $\mathrm{mm}$ (material médio) e 7,51\% dos grãos possuem diâmetro inferior a $0,074 \mathrm{~mm}$. Estes resultados mostram que o argissolo coletado não atende ao exigido pela norma que estabelece que os solos utilizados para tijolos de solo/cimento devem conter entre 10 e $50 \%$ de silte (grãos com diâmetro abaixo de $0,074 \mathrm{~mm}$ ). A argamassa reciclável apresentou $88,73 \%$ dos grãos retidos entre as peneiras 1,190 e 0,149 $\mathrm{mm}$. Destes, $34,82 \%$ ficou retido na peneira de $0,3 \mathrm{~mm}$, e 53,91\% apresentou diâmetro inferior a $0,074 \mathrm{~mm}$ (peneira no 200). A casca de ovo possui granulometria inferior a $0,074 \mathrm{~mm}$. Portanto, a utilização de argamassa reciclável e casca de ovo promoveu a correção da deficiência de ausência de mterial fino no argissolo.

\section{Perda de massa (umidade)}

O monitoramento da perda de massa foi realizado para verificar o comportamento das diferentes proporções de vinhaça, com os outros materiais, ao longo do tempo. A Figura 2 apresenta os resultados médios obtidos para os 7 primeiros dias de cura. Todos os lotes (A, B, C, D e E) apresentaram porcentagem de desvio-padrão inferior a 2,5\%, estes resultados também foram observados por Albuquerque et al. (2008) onde estudou a resistência a compressão mecânica para tijolos de solo-cimento com fabricados com o montículo do cupim. Ainda, nos lotes com dosagens de água/vinhaça de $25 / 75 \%$ e $50 / 50 \%$ (lotes D e E respectivamente), observou-se um perfil comum de absorção (ganho) de umidade entre o 3 ㅇ e 4으 dias de cura. Tal ocorrido pode ser devido à alta umidade relativa do ar nestes dias. Entretanto, este comportamento não foi observado no lote $C$, que possui o mesmo traço dos lotes $D$ e $E$, mas foi hidratado com $100 \%$ de vinhaça. Este resultado pode indicar a viabilidade do uso da vinhaça sem a adição de água para agir como um impermeabilizante.
Figura 2. Monitoramento da perda de massa (gramas) durante os primeiros 7 dias de cura.

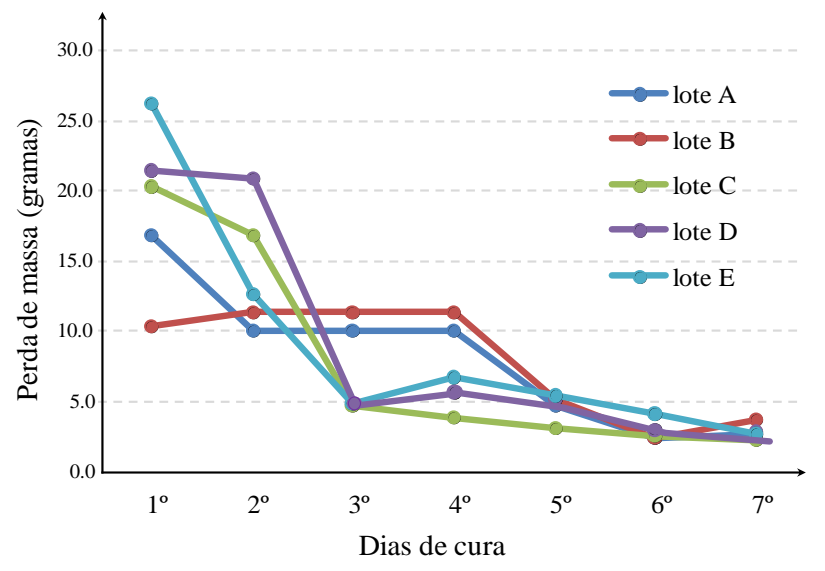

Fonte: Os autores.

A Tabela 2 apresenta as porcentagens, da média, de perda de massa para 7 e 14 dias dos lotes estudados. O monitoramento diário da massa dos cps mostraram que, após o 70 dia de cura a perda diária é inferior a $1 \%$, e no intervalo de 7 a 14 dias a perda de massa máxima foi do lote $E(4,4 \%)$ que possui $50 \%$ de água e $50 \%$ de vinhaça.

Tabela 2. Perda de massa média para 7 e 14 dias.

\begin{tabular}{crr}
\hline \multicolumn{3}{c}{ Perda de massa (\%) } \\
Lotes & 7 dias & 14 dias \\
\hline A & 8,6 & 9,4 \\
\hline B & 7,3 & 8,3 \\
\hline C & 7,9 & 10,5 \\
\hline D & 9,2 & 12,3 \\
\hline E & 6,2 & 10,6 \\
\hline
\end{tabular}

Fonte: Os autores.

\section{Compressão mecânica}

A norma técnica para tijolos de solocimento (ABNT NBR-8491, 2012) estabelece que cada unidade experimental (cp) não deve apresentar valor abaixo de 1,70 MPa e o lote, com mínimo de 3 peças, valores de média inferior a 2,0 MPa para tijolos com 7 dias de cura. Os resultados mostrados na Tabela 3 são referentes aos valores médios da resistência a compressão mecânica (MPa) para 7 e 14 dias de cura. 
Tabela 3. Compressão mecânica dos adobes curados com 7 e 14 dias

\begin{tabular}{crr}
\hline \multicolumn{3}{c}{ Compressão mecânica (MPa) } \\
Lotes & $\mathbf{7}$ dias & 14 dias \\
\hline A & 2,52 & 3,23 \\
\hline B & 1,90 & 2,2 \\
\hline C & 2,03 & 2,25 \\
\hline D & 1,46 & 1,76 \\
\hline E & 1,57 & 1,92 \\
\hline
\end{tabular}

Nos traços A e B fabricados com argissolo, observou-se que a substituição da água pela vinhaça promoveu, nos tijolos do tipo solocimento, curados por 7 dias, $25 \%$ de diminuição nos valores de resistência a compressão mecânica (2,52 $\mathrm{MPa}$ para 1,78 $\mathrm{MPa})$, o que não impossibilita a utilização do argissolo com vinhaça como matriz para a fabricação do tijolo ecológico sobretudo, se estes forem reforçados com outros materiais.

A substituição do cimento pela argamassa reciclável, casca de ovo e diferentes porcentagens de água/vinhaça promoveu a diminuição nos valores de compressão mecânica para os lotes $D$ e $E$, ficando os valores obtidos abaixo de 2,0 MPa exigidos pela NBR. Por outro lado, o lote $C$, que foi fabricação com $100 \%$ de vinhaça, apresentou valor médio de 2,03 $\mathrm{MPa}$ e valores individuais de seus cps superiores a 1,70 $\mathrm{MPa}$, indicando a viabilidade na fabricação de tijolos de adobe deste traço.

\section{CONSIDERAÇÕES FINAIS}

Com as informações obtidas no estudo da fabricação e caracterização física dos tijolos ecológicos a partir de resíduos industriais (casca de ovo, reboco reciclável e vinhaça) vislumbra-se aplicação em habitações sustentáveis. Pelos resultados obtidos pode-se concluir que há viabilidade na fabricação dos tijolos ecológicos com os materiais recicláveis argamassa reciclável, casca de ovo e vinhaça.

Diante dos diferentes traços estudados no processo de fabricação, observou-se que a presença dos materiais recicláveis, em proporções adequadas, favorece o aumento da resistência à compressão mecânica. A substituição da água pela vinhaça nos traços de argissolo diminui sensivelmente os valores de compressão mecânica quando comparados a resistência dos materiais convencionais. Portanto, a vinhaça se mostrou eficaz como agente hidratante dos materiais secos. Os tijolos ecológicos fabricados com argissolo apresentaram resultados satisfatórios de compatibilidade granulométrica, compressão mecânica e perda de água.

\section{AGRADECIMENTOS}

Empresa G10-concreto apoio tecnológico. CNPq apoio a fomento.

\section{REFERÊNCIAS}

ALBUQUERQUE, L. Q. C. de; BISCARO, G. A.; NEGRO, S. R. L.; OLIVEIRA, A. C.de; CARVALHO, L. A.de.; LEAL, S. T. Resistência a compressão de tijolos de solo-cimento fabricados com o montículo do cupim Cornitermes cumulans (Kollar, 1832). Revista Ciência e Agrotecnologia, v. 32, n. 2, p. 553-560, 2008. https://doi.org/10.1590/S1413-

70542008000200032

ABRELPE. ASSOCIAÇÃO BRASILEIRA DE EMPRESAS DE LIMPEZA PÚBLICA E RESÍDUOS ESPECIAIS: Panorama dos resíduos sólidos urbanos no Brasil - 2017/2018. São Paulo: ABLELBE, [2019].

ASSOCIAÇÃO BRASILEIRA DE NORMAS TÉCNICAS. NBR 7181: Solo - Análise granulométrica. Rio de Janeiro, ABNT, 1984.

ASSOCIAÇÃO BRASILEIRA DE NORMAS TÉCNICAS. NBR 8491: Tijolo maciço de solo-cimento. Rio de Janeiro: ABNT,1984 e 2012.

ASSOCIAÇÃO BRASILEIRA DE NORMAS TÉCNICAS. NBR 8492: tijolo maciço de solo-cimento Análise dimensional, Determinação da Resistência à Compressão e da Absorção de águaMétodo de ensaio. Rio de Janeiro: ABNT, 2012.

BRASIL. Ministério do Meio Ambiente, Conselho Nacional de Meio Ambiente, CONAMA. Resolução CONAMA no 307, de 05 de julho de 2002.

BRASILEIRO, L. L.; MATOS, J. M. E. Revisão bibliográfica: reutilização de resíduos da construção e demolição na indústria da construção civil. Revista Cerâmica, v. 61, p. 178189, 2015. https://doi.org/10.1590/0366$\underline{69132015613581860}$

DIAS, R. Gestão ambiental: responsabilidade social e sustentabilidade. São Paulo: Atlas, 2017a. 
DIAS, N. Permacultura para casas e organizações ecológicas, guia prático para economia de recursos e projetos sustentáveis. Instituto Pindorama. Rio de Janeiro: Vietnã Gráfica, 2017b.

FERNANDEZ, J. A. B. Diagnóstico dos resíduos da construção civil: relatório de pesquisa. São Paulo: IPEA, 2012.

GRILLO, C. C.; SARON, C. Wood-plastic from Pennisetum Purpureum Fibers and Recycled Lowdensity Polyethylene. Journal of Natural Fibers, 2020.

https://doi.org/10.1080/15440478.2020.1764436

KLEPA, R. B.; MEDEIROS, M. F.; FRANCO, M. A. C.; TAMBERG, E. T.; FARIAS, T. M. B.; PASCHOALIN FILHO, J. A.; BERSSANETI, F. T.; SANTANA, J. C. C. Reuse of construction waste to produce thermoluminescent sensor for use in highway traffic control. Journal of Cleaner Production, v. 209, p. 250-258, 2019. https://doi.org/10.1016/i.jclepro.2018.10.225

MARQUES, H. F.; RIBEIRO, C.C.; OLIVEIRA, D.M.; BAMBERG, P.; ALMEIDA, M.L.B. Reaproveitamento de resíduos da construção civil: a prática de uma usina de reciclagem no estado do Paraná. Braz. J. of Develop., v. 6, n. 4, p. 21912-21930, 2020. https://doi.org/10.34117/bjdv6n4-383

MENEGAKI, M.; DAMIGOS, D. A review on current situation and challeng of constructionand demolition waste management. Current Opinion in Green and Sustainable. Chemistry, v. 13, p. 815, 2018.

https://doi.org/10.1016/j.cogsc.2018.02.010

MILBRADTI, B. G.; MÜLLER, A. L. H.; SILVA, J. S.; LUNARDI, J. R.; MILANI, L. I. G.; FLORES, É. M. de M.; CALLEGARO, M. G.K.; EMANUELLI, T. Casca de ovo como fonte de cálcio para humanos: composição mineral e análise microbiológica. Revista Ciência Rural, v. 45, n. 3, p. 560-566, 2015. https://doi.org/10.1590/01038478cr20140532

MONTEIRO, J. H. P.; FIGUEIREDO, C. E. M.; MAGALHÃES, A. F.; MELO, M. A. F.; BRITO, J. C. X.; ALMEIDA, T. P. F.; MANSUR, G. L. Manual de gerenciamento integrado de resíduos sólidos. Rio de Janeiro: IBAM, 2001. 200 p.
MOTA, D. N. P.; OLIVEIRA, D. M.; ROCHA, D. J.; NUNES, G. M. P.; SANTOS, G. M. S.; PEREIRA, R. W.; FREIRE, S. H. J.; DINIZ, S. J. S.; MADELLA, U. G. S. Aprimoramento do tijolo adobe com adição de fibras da cana-de-açúcar. R. gest. sust. ambient., v. 9, p. 253-264, 2020. https://doi.org/10.19177/rgsa.v9e02020253-264

NEVES, E. L. B.; GONÇALVES, M. R. F.; CUNHA, E. G.; SEIXAS, J. N. Desempenho térmico de blocos de vedação sustentáveis, compostos de argamassa de cimento e areia e insertos de garrafas PET preenchidas com cinza da casca de arroz. Journal of Experimental Techniques and Instrumentation, v. 4, v. 1, p. 67-78, 2020. https://doi.org/10.30609/jeti.v4i01.11254

NEVES, M. A.Alternativas para valorização da casca de ovo como complemento alimentar e em implantes ósseos. 1998. Dissertação (Mestrado em Ciência dos Alimentos) Departamento de Ciência e Tecnologia de Alimentos, Universidade Federal de Santa Catarina, Florianópolis, Santa Catarina, 1998.

REIS, G. D.; NEGREIROS, N. F.; CANETOMI, T. H. Uso de resíduos da construção civil na fabricação de tijolos solo-cimento. Revista ANAP-Brasil, v. 9, n 6, 2016. https://doi.org/10.17271/1984324091620161440

ROSSETTO, A. J. Utilização agronômica dos subprodutos e resíduos da indústria açucareira e alcooleira. In: PARANHOS, S. B. (ed.) Cana-deaçúcar: cultivo e utilização. Campinas: Fundação Cargill, 1987. v. 2, p. 435-504.

SEGANTINI, A. A. S. Utilização de solo-cimento plástico em estacas escavadas com trado mecânico em llha Solteira-SP. 2000.Tese (Doutorado) - UNICAMP, Campinas, 2000. .

SHARKAWI, A. E. D. M.; ALMOFTY, S. E. D. M.; ABBASS, E. S. M. Performance of green aggregate produced by recycling demolition construction wastes (case study of Tanta City). Engineering, v. 8, n. 2, p. 52-59, 2016. https://doi.org/10.4236/eng.2016.82006

SILVA, T. D.; PAULA, H. M.; SILVA, D.; CARVALHO, I. M.; FONTE, J. T.; PEREIRA, R. R. Uso de granulado de borracha em substituição parcial ao agregado miúdo na produção de tijolos ecológicos. Revista Matéria, v. 22, n. 4, 2017. 
https://doi.org/10.1590/s1517-

$\underline{707620170004.0239}$

SILVA, M. A. S. da; GRIEBELER, N. P.; BORGEET, L. C. Uso de vinhaça e impactos nas propriedades do solo e lençol freático, Revista Brasileira de Engenharia Agrícola e Ambiental, v. 11, n. 1, p. 108-114, 2007. https://doi.org/10.1590/S1415$\underline{43662007000100014}$

SOUZA, M. I. B. Tijolos prensados de solo-cimento confeccionados com resíduos de concreto. Revista Brasileira de Engenharia Agrícola e Ambiental, v. 12, n. 2, p. 205-212, 2008. https://doi.org/10.1590/S1415-

$\underline{43662008000200014}$

TAM, V. W. Y., SOOMRO, M., EVANGELISTA, A. J. A review of recycled aggregate in concrete applications (2000-2017). Construction \& Building Materials, v. 172, p. 272-292, 2018. https://doi.org/10.1016/i.conbuildmat.2018.03.2 $\underline{40}$

TOSELLO, M. E. C.; TAMASHIRO, J. R.; SILVA, L. H. P.; ANTUNES, P. A.; SIMÕES, R. D. Influência de materiais recicláveis e vinhaça da cana de açúcar na resistência mecânica de tijolos ecológicos. Research, society and development, v. 10, p. e56910212911, 2021.

https://doi.org/10.33448/rsd-v10i2.12911 